Mayer/Mayer

Grundriß der allgemeinen Betriebswirtschaftslehre 


\author{
Professor Dr. Leopold Mayer \\ Dozent Dr. Leopold Mayer
}

\title{
Grundriß der allgemeinen Betriebswirtschaftslehre
}

\author{
Zweite, neubearbeitete Auflage
}

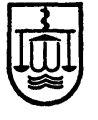

Springer Fachmedien Wiesbaden GmbH 
Copyright by Springer Fachmedien Wiesbaden 1970

Ursprünglich erschienen bei Betriebswirtschaftlicher Verlag Dr. Th. Gabler GmbH - Wiesbaden 1970 Softcover reprint of the hardcover 2nd edition 1970 


\section{Vorwort zur zweiten Auflage}

In der vorliegenden Einführung in die Hauptprobleme der allgemeinen Betriebswirtschaftslehre wurde besonderes Gewicht auf möglichst systematische Gliederung und Darstellung des Stoffes gelegt. Ferner wurden, um den erkenntnistheoretischen Wert der Ausführungen zu verstärken, neben den Verhältnissen in Handels- und Industriebetrieben auch diejenigen in anderen Wirtschaftszweigen fallweise berücksichtigt und soweit als möglich geeignete statistische Daten in den Text eingefügt. Inhaltlich wurden im Hinblick auf die besondere einzel- und gesamtwirtschaftliche Bedeutung das Finanzierungs- und das Kostenproblem in etwas breiterem Umfang erörtert; ferner wurde der Einfluß der Besteuerung und der Wirtschaftsordnung (d. h. die unterschiedliche Entwicklung bei freier und bei gelenkter bzw. gebundener Wirtschaft) auf den Betriebsaufbau und den Betriebsablauf berücksichtigt. Soweit die örtlichen Gegebenheiten (z. B. die Rechtsverhältnisse) für das betriebswirtschaftliche Geschehen mitbestimmend sind, wurden neben den Verhältnissen in der Bundesrepublik Deutschland auch diejenigen in Osterreich in die Untersuchungen einbezogen. Die vorliegende Neuauflage wurde durch Berücksichtigung der seit dem Erscheinen der ersten Ausgabe gewonnenen Erkenntnisse und Entwicklungen ergänzt und erweitert. In den Schrifttumsnachweis sind - abgesehen von einigen grundlegenden theoretischen Arbeiten vor 1945 - vor allem die in den beiden letzten Jahrzehnten erschienenen Veröffentlichungen in Auswahl aufgenommen worden. 


\section{Inhaltsverzeichnis}

Seite

I. Begriff, Aufbau und Entwicklung der Betriebswirtschaftslehre . . 15

A. Wirtschaft und Wirtschaftswissenschaften . . . . . . . . . 15

B. Betriebswirtschaftslehre . . . . . . . . . . . . . . . 15

C. Entwicklung der Betriebswirtschaftslehre . . . . . . . . . . 16

D. Gliederung der Betriebswirtschaftslehre . . . . . . . . . . . 18

II. Unternehmen und Betrieb . . . . . . . . . . . . . . . . . . . 20

III. Allgemeiner Aufbau der Unternehmen und Betriebe . . . . . . . 21

A. Betriebswirtschaft und Wirtschaftsordnung . . . . . . . . . 21

B. Wirtschaftsgrundsätze der Unternehmen bzw. Betriebe (Geschäftsprinzipien) . . . . . . . . . . . . . 21

C. Arten der Betriebe . . . . . . . . . . . . . . . . . . . . . 22

1. Gliederung nach dem Betriebszweck . . . . . . . . . . 22

2. Gliederung nach der Betriebsgröße . . . . . . . . . . . 24

3. Gliederung nach der Kostenstruktur . . . . . . . . . . . 27

4. Gliederung nach der Stellung innerhalb des Unternehmens 28

5. Gliederung nach betrieblichen Hauptfunktionen

(Schwergewicht der Betriebstätigkeit) . . . . . . . . . . 28

D. Unternehmensformen . . . . . . . . . . . . . . . . . 29

1. Ubersicht ... . . . . . . . . . . . . . . . 29

a) Wirtschaftliche Einteilungsgründe . . . . . . . . . . . 29

b) Rechtliche Einteilungsgründe . . . . . . . . . . . . . 29

c) Steuerliche Einteilungsgründe . . . . . . . . . . . . . 32

2. Bestimmungsgründe für die Wahl der Unternehmensform . 33

a) Persönliche Verhältnisse . . . . . . . . . . . . . . . 33

b) Wirtschaftliche Verhältnisse . . . . . . . . . . . . . 34

c) Rechtliche Bestimmungen . . . . . . . . . . . . . . . 34

d) Steuerliche Erwägungen . . . . . . . . . . . . . . 34

e) Politische Einflüsse . . . . . . . . . . . . . . . . . . 34 
Seite

3. Private und öffentliche Unternehmen . . . . . . . . . . 35

4. Einzelunternehmen . . . . . . . . . . . . . . . . 37

5. Gesellschaftsunternehmen . . . . . . . . . . . . 37

6. Erwerbs- und Wirtschaftsgenossenschaften . . . . . . . . 39

E. Zusammenarbeit und Zusammenschlüsse von Unternehmen . . 40

1. Langfristige vertragliche Beziehungen . . . . . . . . . . 40

2. Verbandsbildungen .. . . . . . . . . . . 41

3. Interessennahmen und Interessengemeinschaften . . . . 41

4. Kartelle .. . . . . . . . . . . . . . . . 4 41

5. Konzerne . . . . . . . . . . . . . . . . . . . . 42

6. Trusts . . . . . . . . . . . . . . . . . . 44

IV. Die betrieblichen Produktionsfaktoren . . . . . . . . . . . . . 45

A. Die Arbeit . . . . . . . . . . . . . . . . . . . . 45

1. Stellung des Menschen im Betrieb . . . . . . . . . . 45

2. Rationelle Arbeitsgestaltung . . . . . . . . . . . . . . . 48

3. Soziale Betriebsgestaltung . . . . . . . . . . . . . . . . 49

4. Lohnsysteme . . . . . . . . . . . . . . . 50

a) Zeitlohn . . . . . . . . . . . . . . . . . . . 51

b) Leistungslohn (Werklohn, Akkordlohn) . . . . . . . 52

c) Prämienlohn . . . . . . . . . . . . . . . . . 53

d) Gewinnbeteiligung . . . . . . . . . . . . . 54

5. Personalverwaltung . . . . . . . . . . . . . 55

6. Personalpolitik . . . . . . . . . . . . . . 55

B. Das Kapital . . . . . . . . . . . . . . . . . . . . . . . . 55

1. Begriff . . . . . . . . . . . . . . . . . 55

2. Die Vermögensstruktur . . . . . . . . . . . . . 57

a) Anlage- und Umlaufvermögen . . . . . . . . . 57

b) Betriebs- und Reservevermögen . . . . . . . . . . 59

c) Geld- und Sachvermögen . . . . . . . . . . . . . . . 59

d) Materielles und immaterielles Vermögen . . . . . . . . 60

3. Der Kapitalaufbau . . . . . . . . . . . . . . . . . . 60

a) Eigen- und Fremdkapital (Beteiligungs- und
Kreditkapital) . . . . . . . . . . . . . . . 60

b) Inländisches und ausländisches Kapital . . . . . . 61 
c) Eigen-(Unternehmer-, Beteiligungs-)kapital . . . . . 62

d) Fremd-(Kredit-)kapital . . . . . . . . . . . . . . 64

e) Kapitalkosten . . . . . . . . . . . . . . . 66

f) Kapitaleinsatz und Umsatz . . . . . . . . . 66

g) Vermögensaufbau und Kapitalbeschaffung . . . . . 67

C. Die Organisation . . . . . . . . . . . . . 67

1. Begriff und Wesen der Organisation ........ 67

2. Elemente der Organisation . . . . . . . . . 68

3. Gliederung der Organisationsaufgaben . . . . . . . 68

a) Unternehmensorganisation ........... 68

b) Betriebsorganisation . . . . . . . . . . . . 68

4. Organisationstypen . . . . . . . . . . 69

a) Personelle Aufteilung der Führungsaufgaben

(Kompetenzsysteme) . . . . . . . . . 70

b) Organisation des Arbeits-(Produktions-)ablaufes . . . . 71

c) Aufteilung der Verantwortung . . . . . . . . 71

d) Anpassung der Organisation an den Betrieb . . . . . . 71

e) Räumliche Verteilung der organisatorischen Tätigkeit . 71

V. Der Betriebsablauf . . . . . . . . . . . . . . 72

A. Die betrieblichen Funktionen . . . . . . . . 72

B. Beschaffung . . . . . . . . . . . 73

1. Ubersicht .................. 73

2. Marktorganisation . . . . . . . . . . . 74

a) Nachrichtenwesen . . . . . . . . . . . . 74

b) Auskunftswesen . . . . . . . . . . . 75

c) Kredite und Kreditmärkte . . . . . . . . . . . 75

d) Warenmärkte . . . . . . . . . . . 80

e) Verkehrsmärkte .............. 81

f) Versicherungsmärkte . . . . . . . . . . . 82

g) Arbeitsmärkte . . . . . . . . . . . . . 82

h) Börsenwesen . . . . . . . . . . . . . . . 82

3. Finanzierung .............. 85

a) Begriff und wirtschaftliche Bedeutung . . . . . . 85

b) Mittel der Finanzierung . . . . . . . . . . 86

c) Formen der Finanzierung . . . . . . . . . . 86

d) Allgemeine Finanzierungsgrundsätze . . . . . . . . 89 
e) Finanzplanung . . . . . . . . . . . . . . . . . . . 91

f) Das Liquiditätsproblem . . . . . . . . . . . . . . . 91

g) Effekten als Mittel der Finanzierung . . . . . . . . 93

h) Emissionswesen . . . . . . . . . . . . . . . . 97

i) Finanzierungssonderfälle . . . . . . . . . . . . 98

4. Einkauf und Lagerhaltung . . . . . . . . . . . . . 102

5. Investitionen . . . . . . . . . . . . . 106

C. Fertigung . . . . . . . . . . . . . 112

1. Standortverhältnisse . . . . . . . . . . . . . 112

2. Betriebssysteme . . . . . . . . . . . . . . . 113

a) Handwerk . . . . . . . . . . . . . . . . . . . . 113

b) Verlagswesen (Hausindustrie, Heimarbeit) . . . . . . . 114

c) Manufaktur . . . . . . . . . . . . . . . . . . . . . 114

d) Fabrik . . . . . . . . . . . . . . . . . . . 114

3. Produktionsprozeß . . . . . . . . . . . . . 115

a) Produktionsprogramm . . . . . . . . . . . . 115

b) Produktionsstufen . . . . . . . . . . . . . 115

4. Produktionsweise . . . . . . . . . . . . 116

5. Produktionsorganisation ... . . . . . . . . 117

a) Ablauf des technischen Produktionsprozesses . . . . . . 117

b) Normung, Typung und Spezialisierung . . . . . . 117

c) Arbeitsteilung . . . . . . . . . . . . . 118

d) Verbundwirtschaft . . . . . . . . . . . . . . . . 119

e) Optimalgrößen . . . . . . . . . . . . . . . . . . . . . . 119

6. Produktionsplanung . . . . . . . . . . . . 121

D. Vertrieb . . . . . . . . . . . . . . . . 121

1. Wirtschaftliche Bedeutung . . . . . . . . . . 121

2. Absatz und Umsatz . . . . . . . . . . . . . . . 122

3. Vertriebsorganisation . . . . . . . . . . . 124

a) Utbersicht . . . . . . . . . . . . . . . . . 124

b) Direkter Absatz . . . . . . . . . . . . . . 127

c) Indirekter Absatz . . . . . . . . . . . . . . . 128

4. Bedingtheiten und Grenzen des Absatzes . . . . . . . . 131

a) Marktverhältnisse . . . . . . . . . . . . . . 131

b) Transportverhältnisse . . . . . . . . . . . . 133

5. Marktforschung . . . . . . . . . . . . . . . 133

a) Konjunkturbeobachtung . . . . . . . . . . . . . . . 133

b) Marktbeobachtung . . . . . . . . . . . . . . 134

c) Marktanalyse . . . . . . . . . . . . . . . 134 
6. Absatzplanung . . . . . . . . . . . . 134

7. Werbung ................. 135

E. Unternehmensführung und Verwaltung . . . . . . . 136

VI. Kosten, Wert und Preis ． . . . . . . . . . . . . . . . . . . . 139

A. Begriff der Kosten, Aufwendungen und Ausgaben . . . . . 139

B. Die Kostengliederung . . . . . . . . . . . . . . . . 141

1. Kalkulatorische Kostenelemente . . . . . . . . . . 141

a) Unternehmerlohn . . . . . . . . . . . . . . . 141

b) Anlagenabschreibungen . . . . . . . . . . . . . . 142

c) Kapitalverzinsung . . . . . . . . . . . . . . . . . . 145

d) Wagniskosten . . . . . . . . . . . . . . . 148

e) Sonstige kalkulatorische Kosten . . . . . . . . . . . 151

2. Verrechenbarkeit der Kosten . . . . . . . . . . 151

3. Betriebliche Entstehungsursache . . . . . . . . . 152

4. Häufigkeit des Kostenanfalles . . . . . . . . . . . 153

5. Abhängigkeit der Kosten vom Beschäftigungsgrad . . . . 154

6. Kostenarten . . . . . . . . . . . . . . . . . . 158

a) Materialkosten . . . . . . . . . . . . . . . . . 159

b) Arbeitskosten . . . . . . . . . . . . . . . . . . 161

c) Kapital-, Anlage- und Wagniskosten . . . . . . . 163

d) Fremdleistungskosten . . . . . . . . . . . . . . . . 167

e) Steuern, Gebühren, Beiträge . . . . . . . . . . . 168

7. Kontrollierbarkeit der Kosten . . . . . . . . . . 168

C. Das Wertproblem . . . . . . . . . . . . 169

1. Bewertungsmaßstäbe . . . . . . . . . . . 169

a) Kostenwerte . . . . . . . . . . . . . . . . . . . . . 170

b) Marktwerte (Einzelbewertung) . . . . . . . . . . . . 170

c) Betriebliche Gesamtwerte und davon abgeleitete

Einzelwerte . . . . . . . . . . . 171

d) Schätzungswerte . . . . . . . . . . . . . . . . . 173

e) Buch- und Verrechnungswerte . . . . . . . . . 173

f) Festwerte . . . . . . . . . . . . . . . . . 173

2. Wertfestsetzungsmethoden . . . . . . . . . . 174

3. Bewertungspolitik . . . . . . . . . . . 177

D. Der Preis . . . . . . . . . . . . . . . . . 178

1. Allgemeine Preisbestimmungsfaktoren . . . . . . . 178

2. Arten der Preisbildung . . . . . . . . . . . 180

a) Freie Marktpreise . . . . . . . . . . . . . . . . . . 180

b) Gelenkte und gebundene Preise . . . . . . . . . . . 181 
3. Kostengestaltung und Preispolitik . . . . . . . . . . . 184

4. Kosten, Preise und Erfolg . . . . . . . . . . . . . . . . 186

a) Ertragsteigerung durch Kostensenkung . . . . . . . 187

b) Ertragsteigerung durch Preissenkung . . . . . . . . 187

c) Ertragsteigerung durch Preiserhöhung . . . . . . . . 188

5. Die betriebswirtschaftlichen Kosten- und Ertragsgesetze . . 188

a) Verhältnis Kosten und Preise . . . . . . . . . . . . . 188

b) Verhältnis Kosten und Beschäftigungsgrad . . . . . . 189

c) Aufbau der betrieblichen Kostenstruktur . . . . . . 189

VII. Das Ergebnis (der Erfolg) . . . . . . . . . . . . . 190

A. Begriff . . . . . . . . . . . . . 190

B. Die Abgrenzung des Periodenerfolgs . . . . . . . . . . . . 191

1. Zeitliche Rechnungsabgrenzung und Kostenzurechnung . . 191

2. Ausschaltung von Bewertungseinflüssen . . . . . . 191

3. Ausschaltung von Scheingewinnen . . . . . . . . . 192

C. Die Analyse des Unternehmenserfolgs . . . . . . . . . . 192

D. Rentabilität und Wirtschaftlichkeit des Unternehmens und des

Betriebs ... . . . . . . . . . . 195

1. Rentabilitätsrechnung . . . . . . . . . . 195

2. Wirtschaftlichkeitsmessung . . . . . . . . . 196

a) Kennzahlen auf Grund von Wertgrößen . . . . . . . 196

b) Kennzahlen auf Grund von Mengengrößen . . . . . . 197

E. Einfluß der Besteuerung auf den Erfolg . . . . . . . . . . . 197

F. Funktion und Verwendung des Erfolgs . . . . . . . . . 199

1. Funktion des Erfolgs bei freier und gebundener Wirtschaft 199

2. Gewinnverwendung und Verlustdeckung . . . . . . 199

VIII. Betriebliches Rechnungswesen . . . . . . . . . . 202

A. Einführung und thersicht . . . . . . . . . . 202

1. Grundzwecke des Rechnungswesens . . . . . . . . 202

2. Grundformen des Rechnungswesens . . . . . . . . . . 202

a) Buchhaltung und Bilanz (Zeitrechnung) . . . . . . . 202

b) Kostenrechnung oder Kalkulation (Stückrechnung) . . . 203

c) Betriebsstatistik (Vergleichsrechnung) . . . . . . . . . 203

d) Planung (Vorschau, Budgetierung) . . . . . . . . . 203

3. Vereinheitlichungs-(Normungs-)bestrebungen . . . . . . . 204

B. Buchhaltung . . . . . . . . . . . . . . . 206

1. Gesetzliche Vorschriften . . . . . . . . . . 206 
2. Buchhaltungssysteme . . . . . . . . . . . . . 206

a) Einfache Buchhaltung . . . . . . . . . . . . . . . 206

b) Doppelte Buchhaltung (Doppik) . . . . . . . . . . . . 207

c) Kameralistische Buchhaltung . . . . . . . . . . . . . 208

3. Technik der doppelten Buchhaltung . . . . . . . . . . . 208

a) Formen und Verfahren . . . . . . . . . . . . . . . . 208

b) Kontenrahmen und Kontenplan . . . . . . . . . . . . 214

C. Bilanzen . . . . . . . . . . . . . . . . . . . 215

1. Begriff .. . . . . . . . . . . . . . . . . 215

2. Bilanztypen . . . . . . . . . . . . . . . . . . 216

a) Bilanzzweck . . . . . . . . . . . . . . . . . . . . . 216

b) Bilanzgrundlagen . . . . . . . . . . . . . . . . . 216

c) Bilanztermin . . . . . . . . . . . . . . . . . . . . . 216

d) Bilanzumfang . . . . . . . . . . . . . . . . . . . . 217

e) Unternehmensform und Betriebsgegenstand . . . . . . 217

f) Unternehmensgröße . . . . . . . . . . . . . . . . . 217

3. Allgemeine Bilanzerfordernisse . . . . . . . . . . . . . 218

a) Ordnungsmäßigkeit . . . . . . . . . . . . . . . . . . 218

b) Klarheit und Utbersichtlichkeit . . . . . . . . . . . . 218

c) Wahrheit . . . . . . . . . . . . . . . . . . . . . . 218

d) Vergleichbarkeit . . . . . . . . . . . . . . . . . 219

e) Vollständigkeit . . . . . . . . . . . . . . . . . . . . 219

f) Bilanzdelikte .. . . . . . . . . . . . . . . . . 220

4. Bilanzierungszeitpunkt . . . . . . . . . . . . . 221

a) Bilanzstichtag . . . . . . . . . . . . . . . . . . . . 221

b) Bilanzierungszeitraum . . . . . . . . . . . . . . 222

c) Zeitpunkt der Bilanzfertigstellung . . . . . . . . . . 222

5. Der formelle Aufbau der Vermögens- und Kapitalrechnung 223

a) Allgemeiner Aufbau . . . . . . . . . . . . . . . . . 223

b) Bilanzformblätter . . . . . . . . . . . . . . . . . 223

c) Bilanzsummen . . . . . . . . . . . . . . . . . . . . 224

6. Das Bewertungsproblem . . . . . . . . . . . . . . 224

a) Allgemeine Bedeutung . . . . . . . . . . . . . . 224

b) Bewertungsmaßstäbe .. . . . . . . . . . . . . 224

c) Bewertungsgrundsätze . . . . . . . . . . . . . . . . 224

d) Die Bewertungspraxis . . . . . . . . . . . . . . . . 227

e) Die aktienrechtlichen Bewertungsvorschriften

(§§ 153 bis 156 AktG) . . . . . . . . . . . . . . . . . 227

7. Bilanzauffassungen (Bilanztheorien) . . . . . . . . . . 229 
8. Die Erfolgsrechnung . . . . . . . . . . . . 232

a) Gesamterfolgsrechnung . . . . . . . . . . . . . . . 232

b) Jahreserfolgsrechnung . . . . . . . . . . . . . . . . 232

c) Kurzfristige Erfolgsrechnung . . . . . . . . . . 235

9. Handelsbilanz und Steuerbilanz . . . . . . . . . . 236

a) Steuerbilanzen nach Einkommen-(Körperschaft-)steuerrecht . . . . . . . . . . . . 236

b) Steuerbilanzen nach Vermögensteuerrecht . . . . . . 237

c) Gewerbekapital und Gewerbeertrag . . . . . . . . 238

10. Bilanz- und Betriebsanalyse . . . . . . . . . . . . . 238

a) Bilanzanalyse . . . . . . . . . . . . . . . 238

b) Betriebsanalyse . . . . . . . . . . . . . . . 239

D. Kostenrechnung . . . . . . . . . . . . . . 239

1. Begriff und Zielsetzungen . . . . . . . . . . . . . . 239

2. Arten der Kostenrechnung . . . . . . . . . . . 240

a) Erstellungszeitpunkt . . . . . . . . . . . . . . . 240

b) Aufstellungszweck . . . . . . . . . . . . . . . . . . 240

c) Ermittlungsgrundlagen . . . . . . . . . . . . 240

d) Differenzierung der Kostenträger . . . . . . . . . . . 240

e) Kalkulationsverfahren . . . . . . . . . . . . . . . . 241

3. Betriebsabrechnung . . . . . . . . . . . . . . 245

4. Kostenrechnungssysteme . . . . . . . . . . . . . . . 251

a) Istkostenrechnung zu Vollkosten . . . . . . . . . . 251

b) Istkostenrechnung zu Teilkosten (Grenzkosten) . . . . 251

c) Normalkostenrechnung und Plan- oder Standardkostenrechnung .............. . . 253

Verzeichnis der Abkürzungen . . . . . . . . . . . . . . . . 255

Schrifttumsnachweis . . . . . . . . . . . . . 257

Stichwortverzeichnis ................. 263 